\title{
Effects of complex training versus heavy resistance training on neuromuscular adaptation, running economy and 5-km performance in well-trained distance runners
}

\author{
Fei Li ${ }^{1}$, Ran Wang ${ }^{1}$, Robert U Newton ${ }^{2}$, David Sutton ${ }^{3}$, Yue Shi ${ }^{1}$, Haiyong Ding ${ }^{\text {Corresp. } 1}$ \\ ${ }^{1}$ School of Physical Education and Sport Training, Shanghai University of Sport, Shanghai, Shanghai, China \\ 2 School of Medical and Health Sciences, Edith Cowan University, Joondalup, Australia \\ 3 Talent identification Center and Research Institute, Shanghai Sports School, Shanghai, Shanghai, China \\ Corresponding Author: Haiyong Ding \\ Email address: dinghaiyong@sus.edu.cn
}

Background: Recently, much attention has been paid to the role of neuromuscular function in longdistance running performance. Complex Training (CT) is a combination training method that alternates between performing heavy resistance exercises and plyometric exercises within one single session, resulting in great improvement in neuromuscular adaptation. The purpose of this study was to compare the effect of CT vs. heavy resistance training (HRT) on strength and power indicators, running economy $(\mathrm{RE})$, and 5-km performance in well-trained male distance runners.

Methods: Twenty-eight well-trained male distance runners (19 - 23 years old, $\mathrm{VO}_{2}$ max:65.78 \pm 4.99 $\mathrm{ml} \cdot \mathrm{kg}^{-1} \cdot \mathrm{min}^{-1}$ ) performed one pre-test consisting of: maximum strength (1RM), counter movement jump (CMJ) height, peak power, a drop jump (DJ), and RE assessments, and blood lactate concentration (BLa) measurement at the speeds from $12-16 \mathrm{~km} \cdot \mathrm{h}^{-1}$, a $50-\mathrm{m}$ sprint, and a $5-\mathrm{km}$ running performance test. They were then divided into 3 groups: complex training group $(C T, n=10)$, that performed complex training and endurance training; heavy resistance training group (HRT, $n=9$ ) that performed heavy strength training and endurance training; and control group (CON, $n=9$ ) that performed strengthendurance training and endurance training. After the 8 weeks training intervention, all participants completed a post-test to investigate the training effects on the parameters measured.

Results: After training intervention, both the CT and HRT groups had improvements in: 1RM strength (16.88\%, $p<0.001 ; 18.80 \%, p<0.001$, respectively), CMJ height $(11.28 \%, p<0.001 ; 8.96 \%, p<0.001$, respectively), $14 \mathrm{~km} \cdot \mathrm{h}^{-1} \operatorname{RE}(-7.68 \%, \mathrm{p}<0.001 ;-4.89 \%, \mathrm{p}=0.009$, respectively), 50-m sprints $(-2.26 \%, \mathrm{p}$ $=0.003 ;-2.14 \%, p=0.007$, respectively) and 5-km running performance $(-2.80 \%, p<0.001 ;-2.09 \%, p$ $<0.001$, respectively). The CON group did not show these improvements. All three training groups showed improvement in the $12 \mathrm{~km} \cdot \mathrm{h}^{-1} \mathrm{RE}(\mathrm{p} \leq 0.01)$. Only the CT group exhibited increases in DJ height (12.94\%, $p<0.001)$, reactive strength index (19.99\%, $p<0.001), 16 \mathrm{~km} \cdot \mathrm{h}^{-1} \mathrm{RE}(-7.38 \%, p<0.001)$, and a reduction of BLa concentrations at the speed of $16 \mathrm{~km} \cdot \mathrm{h}^{-1}(-40.80 \%, p<0.001)$ between pre- and posttests.

Conclusion. This study demonstrated that CT can enhance 1 RM strength, CMJ height, 12 and $14 \mathrm{~km} \cdot \mathrm{h}^{-1}$ REs, 50-m sprints and 5-km running performances in well-trained male distance runners and may be superior to HRT for the development of reactive strength and $16 \mathrm{~km} \cdot \mathrm{h}^{-1} \mathrm{RE}$, and reduction of BLa concentrations at speed of $16 \mathrm{~km} \cdot \mathrm{h}^{-1}$. Young male distance runners could integrate CT into their programs to improve the running performance.

Peer) reviewing PDF | (2018:12:33402:2:0:NEW 5 Mar 2019) 


\section{Effects of complex training versus heavy resistance}

\section{2 training on neuromuscular adaptation, running}

3 economy and 5-km performance in well-trained

\section{4 distance runners}

$5 \quad$ Fei Li ${ }^{1}$, Ran Wang ${ }^{1}$, Robert U Newton ${ }^{2}$, David Sutton ${ }^{3}$, Yue Shi ${ }^{1}$, Haiyong Ding ${ }^{1}$

$6 \quad{ }^{1}$ School of Physical Education and Sport Training, Shanghai University of Sport, Shanghai,

7 China

$8{ }^{2}$ School of Medical and Health Sciences, Edith Cowan University, Joondalup, WA, Australia

$9 \quad{ }^{3}$ Talent identification Center and Research Institute, Shanghai Sports School, Shanghai, China

11 Corresponding Author:

12 Haiyong Ding,

13 School of Physical Education and Sport Training,

14 Shanghai University of Sport, Shanghai, China

15 Email: dinghaiyong@sus.edu.cn

16 Fax: +(86)-21-65507101

17 Telephone: $+(86)-21-65507101$ 


\section{Abstract}

27 Background: Recently, much attention has been paid to the role of neuromuscular function in

28 long-distance running performance. Complex Training (CT) is a combination training method

29 that alternates between performing heavy resistance exercises and plyometric exercises within

30 one single session, resulting in great improvement in neuromuscular adaptation. The purpose of

31 this study was to compare the effect of CT vs. heavy resistance training (HRT) on strength and

32 power indicators, running economy (RE), and 5-km performance in well-trained male distance

33 runners.

34 Methods: Twenty-eight well-trained male distance runners (19 - 23 years old,

$35 \mathrm{VO}_{2} \mathrm{max}: 65.78 \pm 4.99 \mathrm{ml} \cdot \mathrm{kg}^{-1} \cdot \mathrm{min}^{-1}$ ) performed one pre-test consisting of: maximum strength

36 (1RM), counter movement jump (CMJ) height, peak power, a drop jump (DJ), and RE

37 assessments, and blood lactate concentration (BLa) measurement at the speeds from 12 - 16

$38 \mathrm{~km} \cdot \mathrm{h}^{-1}$, a 50-m sprint, and a 5-km running performance test. They were then divided into 3

39 groups: complex training group $(\mathrm{CT}, \mathrm{n}=10)$, that performed complex training and endurance

40 training; heavy resistance training group $(\mathrm{HRT}, \mathrm{n}=9)$ that performed heavy strength training and

41 endurance training; and control group $(\mathrm{CON}, \mathrm{n}=9)$ that performed strength-endurance training

42 and endurance training. After the 8 weeks training intervention, all participants completed a post-

43 test to investigate the training effects on the parameters measured.

44 Results: After training intervention, both the CT and HRT groups had improvements in: 1RM

45 strength $(16.88 \%, \mathrm{p}<0.001 ; 18.80 \%, \mathrm{p}<0.001$, respectively $)$, CMJ height $(11.28 \%, \mathrm{p}<0.001$; 
$8.96 \%, \mathrm{p}<0.001$, respectively), $14 \mathrm{~km} \cdot \mathrm{h}^{-1} \mathrm{RE}(-7.68 \%, \mathrm{p}<0.001 ;-4.89 \%, \mathrm{p}=0.009$,

47 respectively), 50-m sprints $(-2.26 \%, \mathrm{p}=0.003 ;-2.14 \%, \mathrm{p}=0.007$, respectively) and $5-\mathrm{km}$

48 running performance $(-2.80 \%, \mathrm{p}<0.001 ;-2.09 \%, \mathrm{p}<0.001$, respectively). The CON group did

49 not show these improvements. All three training groups showed improvement in the $12 \mathrm{~km} \cdot \mathrm{h}^{-1}$

$50 \mathrm{RE}(\mathrm{p} \leq 0.01)$. Only the CT group exhibited increases in DJ height $(12.94 \%, \mathrm{p}<0.001)$, reactive

51 strength index $(19.99 \%, \mathrm{p}<0.001), 16 \mathrm{~km} \cdot \mathrm{h}^{-1} \mathrm{RE}(-7.38 \%, \mathrm{p}<0.001)$, and a reduction of BLa

52 concentrations at the speed of $16 \mathrm{~km} \cdot \mathrm{h}^{-1}(-40.80 \%, \mathrm{p}<0.001)$ between pre- and post-tests.

53 Conclusion. This study demonstrated that CT can enhance 1RM strength, CMJ height, 12 and 14

$54 \mathrm{~km} \cdot \mathrm{h}^{-1} \mathrm{REs}, 50-\mathrm{m}$ sprints and 5-km running performances in well-trained male distance runners

55 and may be superior to HRT for the development of reactive strength and $16 \mathrm{~km} \cdot \mathrm{h}^{-1} \mathrm{RE}$, and

56 reduction of BLa concentrations at speed of $16 \mathrm{~km} \cdot \mathrm{h}^{-1}$. Young male distance runners could

57 integrate $\mathrm{CT}$ into their programs to improve the running performance.

Key Words: reactive strength; strength and power; endurance performance; distance running; 


\section{Introduction}

64 Maximum oxygen uptake $\left(\mathrm{VO}_{2} \mathrm{max}\right)$, lactate threshold (LT), and running economy (RE)

65 have been determined to be the most crucial physiological factors influencing long-distance

66 running performance (Midgley et al. 2007). As running pace increases (Díaz et al. 2018), runners

67 must be able to sustain a relatively high speed over the course of competition, during which the

68 practical energy requirements may surpass the power output of their aerobic system (Paavolainen

69 et al. 2000). Because elite runners share similar levels of $\mathrm{VO}_{2} \mathrm{max}$ and LT (Beattie et al. 2014), it

70 is difficult to use these factors to distinguish the endurance performance. Consequently, in

71 addition to central factors such as cardiovascular capacity, peripheral factors relating to

72 neuromuscular function also play a critical role in endurance performance (Nummela et al. 2006;

73 Paavolainen et al. 2000). In trained endurance runners, chronic strength training elicits increases

74 in RE coincident with improved performance, that is partially due to neuromuscular adaptations

75 associated with strength training (Beattie et al. 2017; Piacentini et al. 2013). Heavy resistance training (HRT) and plyometric training (PLY) are the most common strength training methods

77 utilized by distance runners due to their role in developing the muscle force production and

78 function of the stretch-shortening cycle (SSC), which are critical contributors for distance

79 running (Spurrs et al. 2003; Storen et al. 2008). However, insufficient research has been

80 conducted on the impact of simultaneous heavy strength and plyometric training on endurance

81 performance. 
82 Complex training (CT) is described as a combination training method that alternates

83 between performing heavy resistance exercises and plyometric exercises within one single

84 session (Macdonald et al. 2012). These two types of exercises performed consecutively in CT are

85 referred to as a 'complex pair' that has significant effects on the development of strength and

86 power (one repetition maximum strength [1RM], jump, and sprint performance) in team-sport

87 athletes (Maio Alves et al. 2010; Santos \& Janeira 2008). CT can elicit a post-activation

88 potentiation (PAP) response, thus allowing individuals to produce more power on the subsequent

89 exercise (Carter et al. 2014). The specific mechanisms include stimulating high order motor unit

90 recruitment and excitability, increasing phosphorylation of the myosin light chain, and changes

91 in limb stiffness (Blagrove et al. 2018; Tillin \& Bishop 2009). These neuromuscular adaptations

92 may also benefit long-distance performance such as improvement in RE and maximal running

93 speed (Beattie et al. 2014; Blagrove et al. 2018). In addition, CT is a time-efficient training

94 method allowing distance runners who have limited time for strength training to perform two

95 types of training at the same time. Although CT has consistently been shown to improve

96 performance for team-sport athletes, to the best of our knowledge, the benefit of CT in terms of

97 neuromuscular adaptation, RE and endurance performance has received far less attention.

98 Understanding the role of CT has a practical application for guiding distance runners' training.

99 Therefore, the primary purpose of this study was to investigate the effect of an 8-week CT

100 regimen on neuromuscular adaptation (1RM strength, jumping performance, and reactive

101 strength), running economy, and 5-km running performance in well-trained male runners. As 
102 previous research has already confirmed the impact of HRT on endurance performance

103 (Piacentini et al. 2013; Storen et al. 2008), this study also aimed to compare the effect of CT and

104 HRT. We hypothesized that CT performed concurrently with endurance training will lead to

105 greater neuromuscular adaptations and endurance performance improvements than HRT in well-

106 trained male long-distance runners.

107

108 Methods

109 Experimental Approach to the Problem

110 The current study used a between-group repeated-measure design to test the hypothesis that

111 CT improves neuromuscular adaptations, running economy, and endurance running performance

112 to a greater extent as compared to that with HRT. This study began in the early stage of the

113 winter training block (November, December, and January), lasted for 10 weeks, and was

114 comprised of four parts: 2 weeks of preparatory strength training, a pre-test, 8 weeks of training

115 intervention, and a post-test (Figure 1). After the preparatory strength training, all participants

116 underwent a two-day test protocol to assess body composition, 1RM strength, counter movement

117 jump (CMJ), and drop jump (DJ), running economy, $\mathrm{VO}_{2} \mathrm{max}$, and 50-m and 5-km running

118 performance as the pre-test. They were then divided into three groups matched by age, body

119 composition, $\mathrm{VO}_{2} \mathrm{max}$, and 5-km running time. Groups consisted of: a complex training group,

120 CT $(\mathrm{n}=10)$ who performed complex training and endurance training; a heavy resistance training

121 group, HRT $(\mathrm{n}=9)$ who performed heavy strength training and endurance training; and a control 
122 group, $\mathrm{CON}(\mathrm{n}=9)$ who performed strength-endurance training and endurance training. After

123 the 8-week training intervention, all participants completed a post-test, which was identical to the

124 pre-test.

125

126 Participants

127 Twenty-eight male runners on a collegiate long-distance running team volunteered to

128 participate in this study and their basic physical characteristics, related body composition, and

129 physiological indicators are provided in Table 1. All the participants competed at the collegiate

130 level, they had a minimum of four years of training experience for long-distance running and

131 were free from injury. They stayed and trained together at the training center affiliated with the

132 Shanghai University of Sport, China, thus having similar training, recovery, and nutritional

133 environments. The participants and their coaches were extensively informed about the research

134 process and potential risks associated with the study and subsequently signed an informed

135 consent document before participation. All participants were free from any health problems that

136 would affect physical performance or put them at risk. The study was approved by the Ethics

137 Committee of Shanghai University of Sport, China (ID number:2017047).

\section{Procedures}

140 Preparatory strength training. 
141 During the preparatory strength training phase, all participants were asked to master the

142 correct strength training techniques and familiarize themselves with the testing procedures to

143 ensure training efficacy and testing accuracy. All exercises employed during the 2-week

144 preparatory strength training phase were the same as that in the training intervention except that

145 the load was reduced to a minimum (all participants used an empty $20 \mathrm{~kg}$ barbell). All 3 training

146 groups performed their routine endurance training during this phase.

148 Pre-test and post-test

149 All participants performed a two-day test protocol. They were tested for by body composition,

150 1RM strength, $\mathrm{CMJ}$, and DJ on the first day, and performed $\mathrm{RE}, \mathrm{VO}_{2} \mathrm{max}, 50-\mathrm{m}$ and 5-km

151 running performance tests on the second day. Participants fasted two hours before the test, and

152 wore the same running shoes during the pre- and post-test.

153

154 Body composition test. Height was measured using a wall-mounted stadiometer (Butterfly,

155 Shanghai, China) and recorded to the nearest $0.1 \mathrm{~cm}$. Body mass, fat mass and fat-free mass were

156 measured using a bioimpedance analyzer (X-scan plus II, Jawon, Korea). Body mass index

157 (BMI) was calculated as body mass divided by height squared.

159 Neuromuscular adaptation tests

160 As muscle force and power production and the function of the SSC are important factors for 
161 distance runners' performance (Spurrs et al. 2003; Storen et al. 2008), neuromuscular adaptation

162 was tested using the 1RM strength test, CMJ and DJ test.

163 1RM strength test. This test was performed in a power rack (Hammer Strength, Rosemont,

164 Illinois, USA) using a previously described protocol for measuring 1RM back squat strength

165 (MCBRIDE et al. 1999). Following the general warm up, each subject started with 4 warm-up

166 sets of 10 reps at $50 \%, 5$ reps at $70 \%, 3$ reps at $80 \%$ and 1 rep at $90 \%$ of their estimated $1 \mathrm{RM}$.

167 Each subject's warm-up 1RM load was estimated by the researchers based on their body weight,

168 training experience and age. After the warm-up sets, the subject performed 3 to 4 maximal-effort

169 trials to determine their actual 1RM, with a rest interval of 3 to $5 \mathrm{mins}$. Each subject was asked to

170 squat through a full range of motion by lowering the bar to the point where the thigh was parallel

171 with the ground. Two spotters monitored the participants throughout the test.

172 Counter movement jump. After the 1RM test, each participant rested for 15 mins, and then

173 performed a CMJ and DJ test from a $40 \mathrm{~cm}$ height. Jumping height, peak power (maximum

174 power during $\mathrm{CMJ}$ ), foot contact time, and other related parameters were recorded using a force

175 platform (9290AA, Kistler, Winterthur, Switzerland). To perform the CMJ, the participants were

176 asked to stand on the force platform and place their hands on their hips. The subject then

177 performed a rapidly downward squat movement and jumped vertically to attain maximum

178 height. Arm-swing was not allowed during the jump. Three trials separated by 1 minute of

179 passive recovery were performed. The best trial for jump height was included in the data

180 analysis.

Peer] reviewing PDF | (2018:12:33402:2:0:NEW 5 Mar 2019) 
181 Reactive strength, normally by measured by DJ height and reactive strength index (RSI)

182 (Suchomel et al. 2016), is defined as a runners' capacity to efficiently utilize the SSC and elastic

183 energy produced by the muscle-tendon complex (Beattie et al. 2017). For the DJ test, all

184 participants were asked to stand on a 40cm-high box and place their hands on their hips. The

185 participants then stepped off the box to land on the force plate and jumped vertically for

186 maximum height and minimum ground contact time. The trial was successful only when the

187 participants did not bend the hip or knee during the jump and their hands did not leave the hips.

188 Three trials separated by 1 minute of passive recovery were performed. The best trial for jump

189 height was included in the data analysis. The RSI was calculated by dividing jumping height in

190 cm by contact time in seconds.

191

192 Running economy and related physiological tests

193 All physiological variables $\left(\mathrm{VO}_{2} \mathrm{max}, \mathrm{RE}\right.$, and blood lactate concentration, [BLa]) were

194 measured using the treadmill protocol (Life Fitness T5, Rosemont, Illinois, USA). Oxygen

195 uptake and heart rate (HR) were determined using a portable metabolic analyzer (K5, Cosmed

196 Srl, Rome, Italy) and HR monitor belt (Garmin, Olathe, Kansas, USA). Finger blood, was

197 collected prior to the test, was used to determine if the participant was in a normal state. The

198 subject then warmed-up on the treadmill set to $8 \mathrm{~km} \cdot \mathrm{h}^{-1}$ for $10 \mathrm{mins}$. After the warm-up period,

199 the subject rested for 5 mins and then began a 4-minute run at each of 3 incremental speeds (12,

20014 , and $16 \mathrm{~km} \cdot \mathrm{h}^{-1}$, respectively) to determine RE, which was defined as the average $\mathrm{VO}_{2}\left(\mathrm{ml} \cdot \mathrm{kg}^{-}\right.$ 
$201^{1} \cdot \mathrm{min}^{-1}$ ) data during the last minute of each running speed. This RE testing protocol was similar

202 to that in previous studies (Cole et al. 2006; Sedano et al. 2013) and reflected the runners' ability

203 to run at submaximal speeds. Participants ran for 4 minutes to ensure adequate time for their

$204 \mathrm{VO}_{2}, \mathrm{HR}$, and BLa to reach a steady-state (Beattie et al. 2017; Saunders et al. 2006). After each

205 4-minute stage of running, the subject rested for $1 \mathrm{~min}$, during which finger blood samples were

206 collected. All finger blood samples were used to measure their blood lactate concentrations and

207 aerobic capacity during running via a lactate analyzer (EKF Diagnostic, Magdeburg, Germany).

208 After the completion of the last stage of the running economy test, the treadmill speed was

209 set to $17 \mathrm{~km} \cdot \mathrm{h}^{-1}$, which was then increased by $1 \mathrm{~km} \cdot \mathrm{h}^{-1}$ every $2 \mathrm{mins}$, until the subject reached

210 exhaustion. The following criteria were used to determine exhaustion: heart rate greater than

$21190 \%$ of age-predicted maximal HR (calculated by 220 - runner's age); respiratory exchange ratio

$212(\mathrm{RER}) \geq 1.10$; and rating of perceived exertion (RPE) above $18 . \mathrm{VO}_{2}$ max was determined as the

213 highest $\mathrm{VO}_{2}$ value using a 30s moving window.

215 50-m sprint and 5-km running performance test.

216 For testing the runner's maximal speed and endurance performance, the 50-m and 5-km running

217 tests were performed on a $200-\mathrm{m}$ indoor track with an ambient temperature of $20-22^{\circ} \mathrm{C}$ and a

218 relative humidity of $61-64 \%$. Following a 15-min warm-up, the participants performed three

219 50-m maximal effort sprint trials with a standing start position. The sprint time was measured via

220 two timing gates (Smart Speed, Fusion Sport, Australia) and the best trial for sprint time was 
221 included in the data analysis. After a 10-mins rest, for ease and accuracy when measuring the

222 trial time, the participants were randomly divided into 4 groups of 7 participants, and instructed

223 to finish the 5-km running test as fast as possible. Verbal encouragement was provided

224 throughout the test. The trial time was recorded using a stopwatch (Tianfu, Shenzhen,

225 Guangdong, China).

226

227

\section{Training Intervention}

228

During the training intervention, the participants were asked to perform 9 training sessions

229

per week (6 endurance and 3 strength sessions) with the schedule provided in Table 2.

230

Participants performed different strength training programs according to their group allocation

231 (Table 3). Participants were asked to avoided any other strength training besides the training

232 intervention. All strength sessions were supervised by certified strength and conditioning

233 specialists.

234

235 Complex training group. This group performed 3 sets of 3 complex pairs (the pairing of two

236 biomechanically similar exercises), including a back squat + a DJ from a $40 \mathrm{~cm}$ box (pair 1); a

237 Bulgarian squat + a single leg hop (pair 2); and a Romanian deadlift + a double leg $50 \mathrm{~cm}$ hurdle

238 hop (pair 3). All of the strength training performed in the complex pairs involved heavy loads

$239(80-85 \% 1 \mathrm{RM})$ based on the pre-1RM strength test to enhance the power output of the

240 subsequent plyometric exercises(Carter et al. 2014). The intra-complex rest interval (ICRI) was 
241 set at 4 mins since this is the optimal time for exploiting power according to previous

242 studies(Comyns et al. 2006).

243

244 Heavy resistance training group. This group performed the identical lower limb strength

245 training as the CT group, but excluded the plyometric exercises. In order to ensure that the CT

246 and HRT workloads were similar, the HRT group added two additional sets for each strength

247 exercises (same intensity and repetition) to match the plyometric training load in the CT group.

248 The recovery time between sets was 3 mins as this is the optimal time for improving maximum

249 strength in distance runners. (Storen et al. 2008)

250

251 Control group. This group performed the strength-endurance regimen. The resistance exercises

252 the CON group used were as same as the CT and HRT groups, but the load was reduced to $40 \%$

$2531 \mathrm{RM}$ and the repetitions were increased to $20-30$. In addition, the CON group added two

254 more sets for each strength exercises (same intensity and repetition) to match the plyometric

255 training or heavy resistance training load. The recovery time between sets was 1 min because a

256 muscular strength-endurance training program has very short rest period (Sedano et al. 2013).

258 Endurance training. In addition to strength training, all three groups performed the same

259 endurance training, which mainly consisted of long-distance road running at an intensity of 70 -

$26085 \%$ maximal heart rate $\left(\mathrm{HR}_{\mathrm{MAX}}\right)$ and interval training at an intensity of $90-95 \% \mathrm{HR}_{\mathrm{MAX}}$. The 
261 total endurance training distance was $77.25 \pm 2.33 \mathrm{~km}$ per week, and the total endurance and

262 strength training times were $8.75 \pm 0.97$ and $3.5 \pm 0.5$ hours /week, respectively.

263

264 Statistical Analyses

265 Statistical analyses were performed using SPSS 22.0 (IBM Corp. Armonk, NY, USA).

266 Means \pm standard deviations (SD) are shown in Tables 4 - 6. Normality and homogeneity of

267 variances were tested via Shapiro-Wilk and Levene's tests, respectively. All variables were

268 tested using a one-way analysis of variance (ANOVA) to determine differences among the 3

269 training groups. Two-way repeat measures were performed using ANOVA with time (pre- vs.

270 post-test) and group (CT vs. HRT vs. CON) as factors to assess training-related effects. When a

271 statistically significant difference was found for time by group interaction effects or main effects

$272(\mathrm{p} \leq 0.05)$, pairwise comparisons were performed using a post hoc $\mathrm{t}$ test with Bonferroni

273 correction. For effect size, partial eta-squared was calculated $(\eta 2)$, and $0.2,0.5$, and 0.8 were

274 interpreted as small, medium and large effect sizes, respectively (Cohen 1988). An alpha level of

$275 \mathrm{p}<0.0167$ was set for establishing statistical significance.

276

277 Results

278 All pre- and post-test variables are shown in Tables 4 - 6. No differences were found in any

279 of these variables among the CT, HRT, and CON groups during the pre-test. Body mass, BMI, 
280 fat-free mass, fat mass and fat mass percentages remained unaltered in all 3 groups from pre- to

281 post-test (Table 4).

282

283 Strength and power test

284

The two-way repeated measures ANOVA showed a significant time by group interaction or

285

time main effect on $1 \mathrm{RM}\left(\mathrm{F}_{(2,25)}=17.414, \mathrm{p}<0.001, \eta 2=0.578\right)$, CMJ height $\left(\mathrm{F}_{(2,25)}=7.404\right.$,

286

$\mathrm{p}=0.03, \eta 2=0.372)$, peak power $\left(\mathrm{F}_{(1,25)} 11.589, \mathrm{p}=0.002, \eta 2=0.317\right)$, DJ height $\left(\mathrm{F}_{(2,25)}=\right.$

287

25.355, $\mathrm{p}<0.001, \eta 2=0.670)$, and $\operatorname{RSI}(\mathrm{F}(2,25)=4.914, \mathrm{p}=0.016, \eta 2=0.282)$. The Bonferroni

post hoc test showed that there were significant increases between the pre- and post-tests in:

289

1RM strength (CT: 16.88\%, p < 0.001; HRT: 18.80\%, p < 0.001) and CMJ height (CT: 11.28\%,

290

$\mathrm{p}<0.001$; HRT $: 8.96 \%, \mathrm{p}<0.001$ ), but not for peak power (CT: $8.20 \%, \mathrm{p}=0.02$; HRT: $5.05 \%, \mathrm{p}$

291

$=0.036)$ in the $\mathrm{CT}$ and HRT groups. Only the $\mathrm{CT}$ group showed a significant increase between

292

the pre- and post-test for DJ height $(12.94 \%, \mathrm{p}<0.001)$ and RSI $(19.99 \%, \mathrm{p}<0.001)$. No

significant changes were observed in the CON group for these indicators (Figure 2).

\section{Running economy and related physiological test}

Table 6 demonstrated that there were no significant differences in $\mathrm{VO}_{2} \max$ in the time by

group interaction or main effects after the 8 -week intervention. For the RE test, there was a

significant interaction between time and group on $16 \mathrm{~km} \cdot \mathrm{h}^{-1} \mathrm{RE}\left(\mathrm{F}_{(2,25)}=5.646, \mathrm{p}=0.009, \eta 2=\right.$ 
300 pre- and post-test only in the CT group $(-7.38 \%, \mathrm{p}<0.001)$. There was a significant time main

301 effects for $12 \mathrm{~km} \cdot \mathrm{h}^{-1} \mathrm{RE}\left(\mathrm{F}_{(1,25)}=23.554, \mathrm{p}<0.001, \eta 2=0.485\right)$ and each group had a

302 significant improvement between the pre- and post-test (CT: $-4.47 \%, p=0.005 ; \mathrm{HRT}:-4.11 \%, \mathrm{p}$

$303=0.016$; CON: $-4.38 \%, \mathrm{p}=0.01)$. For the $14 \mathrm{~km} \cdot \mathrm{h}^{-1} \mathrm{RE}$, a significant time main effect $\left(\mathrm{F}_{(1,25)}\right.$

$304=28.9244, \mathrm{p}<0.001, \eta 2=0.536)$ was observed: the CT $(-7.68 \%, \mathrm{p}<0.001)$ and HRT groups $(-$

$3054.89 \%, \mathrm{p}=0.009$ ) showed a significant improvement between the pre- and post-test (Figure 3).

306 In addition, there was a significant time by group interaction in BLa at $16 \mathrm{~km} \cdot \mathrm{h}^{-1}\left(\mathrm{~F}_{(2,25)}=\right.$

$3074.2182, \mathrm{p}=0.026, \eta 2=0.252$ ). The Bonferroni post hoc test identified that the $\mathrm{BLa}$ at $16 \mathrm{~km} \cdot \mathrm{h}^{-1}$

308 was significantly decreased in the CT group $(-40.80 \%, \mathrm{p}<0.001)$ (Figure 4). There were no

309 significant time by group interactions or main effects on HR at any speed after the intervention.

\section{$311 \quad 5-\mathbf{k m}$ running and 50-m sprint performance test}

312 There was a statistically significant time by group interaction in 5-km running performance

$313\left(\mathrm{~F}_{(2,25)}=9.627, \mathrm{p}=0.001, \eta 2=0.435\right)$. The Bonferroni post hoc test showed that there were

314 significant improvements between the pre- and post-tests in the CT $(-2.80 \%, \mathrm{p}<0.001)$ and HRT

$315(-2.09 \%, \mathrm{p}<0.001)$ groups, and the CT group was significantly lower when compared to the

316 CON group at the post-test $(\mathrm{p}=0.001)$. There was a significant time main effects for the $50-\mathrm{m}$

317 sprint performance $\left(\mathrm{F}_{(1,25)}=14.903, \mathrm{p}=0.001, \eta 2=0.373\right)$, and the CT $(-2.26 \%, \mathrm{p}=0.003)$ and

318 HRT groups $(-2.14 \%, \mathrm{p}=0.007)$ showed a significant improvement between the pre- and post- 
319 tests (Figure 5). There were no significant changes in the CON group in terms of 5-km running

320 or $50-\mathrm{m}$ sprint performance after the intervention.

321

322 Discussion

323 The aim of this study was to determine the effects of CT vs. HRT on neuromuscular

324 adaption, RE and 5-km performance in well-trained male distance runners. We hypothesized that

325 CT would elicit greater training benefits than HRT, which was supported by the results of this

326 study. The primary finding of this study was that performing CT or HRT with endurance training

327 significantly increased strength and power performance, RE at 12 and $14 \mathrm{~km} \cdot \mathrm{h}^{-1}, 50-\mathrm{m}$ sprint

328 times, and 5-km performance in well-trained male distance runners. Furthermore, CT

329 demonstrated a greater benefit in terms of reactive strength, $16 \mathrm{~km} \cdot \mathrm{h}^{-1} \mathrm{RE}$ and reduction of BLa

330 concentrations at $16 \mathrm{~km} \cdot \mathrm{h}^{-1}$. Long-distance runners could integrate this time-efficient training

331 approach into their training program for improving running performance.

332

333 Neuromuscular adaptation

334 Neuromuscular adaptations, including 1 RM strength and jumping ability, were especially

335 important indicators of increasing athletic capacity in strength and power athletes, and recent

336 studies showed that they also have a positive impact on modifying distance running performance

337 such as increasing force production during running and increasing RE (Berryman et al. 2017;

338 Stone et al. 2006). After the training intervention, there was a significant increase in 1RM 
339 strength for participants in the CT (16.88\%) and HRT groups (18.80\%), but not in the CON

340 group. In accordance with our results, previous studies have demonstrated similar improvements

341 (12 - 33.2\%) in 1 RM strength after 8 weeks of HRT among distance runners (Damasceno et al.

342 2015; Storen et al. 2008). These results were consistent because the similar heavy load strength

343 programs (i.e. 3 - 5RM) were adopted in previous studies. Heavy strength training exerts high

344 mechanical stimulus on the neuromuscular system, thus producing good effects on maximal

345 strength development. It has been reported that high load strength training (3 - $6 \mathrm{RM})$ is

346 associated with maximal force generation without muscle hypertrophy, and is attributed to neural

347 adaptation (Docherty \& Sporer 2000). This is consistent with our results. Considering that no

348 changes in body weight, fat-free mass, and BMI were observed, we hypothesized that the

349 improved maximum strength likely resulted from neural adaptation such as motor unit

350 recruitment and increase in firing frequency (Cormie et al. 2011). This was a concern for

351 distance runners because increased body mass negatively impacts physiological parameters, such

352 as $\mathrm{VO}_{2}$ max or RE, and inevitably impairs endurance development (Beattie et al. 2017).

353 With respect to power performance, the CT and HRT groups showed a significant increase

354 in CMJ height (11.28\% and 8.96\%, respectively) after the intervention. The CMJ height increase

355 in the CT group was higher than the improvements $(4.5-8.9 \%)$ noted in previous studies which

356 used HRT or PLY training interventions in distance runners with similar intervention timeframes

357 (Ramírezcampillo et al. 2014; Vikmoen et al. 2016). This may be attributed to the different

358 strength training protocols in the training arrangement. Previous research has confirmed the 
359 positive effects of CT on increasing power performance and may be more efficient than using

360 one strength training method (Macdonald et al. 2012). The mechanism underpinning this

361 phenomenon may be attributable to HRT causing increases in motor neuron excitability, thus

362 creating optimal conditions for subsequent plyometric exercises (Esformes \& Bampouras 2013).

363 The cumulative effect of CT would then lead to greater power output (Jones \& Lees 2003).

364 Reactive strength is defined as the ability of the muscle-tendon complex to produce greater

365 force during the concentric contraction immediately following a rapid eccentric contraction

366 (Beattie et al. 2016). These indicators, such as DJ and RSI, are very important for distance

367 running performance (Beattie et al. 2017). In the present study, the CT group showed a

368 significant improvement in DJ performance (12.94\%) and RSI (19.99\%) between the pre- and

369 post-tests, whereas no changes were noted in the HRT and CON groups. The advantageous CT

370 effect on reactive strength can be explained by the inclusion of plyometric exercises such as drop

371 jump, single leg hops, and hurdle hops in CT. These exercises exerted maximal external force on

372 the neuromuscular system in a very short time, greatly enhancing power by developing SSC

373 utilization and leg musculotendinous stiffness (Saunders et al. 2006; Spurrs et al. 2003).

374 Interestingly, our results are also consistent with those of the study by Ramírez-campillo et al.

375 (Ramírezcampillo et al. 2014), who reported improvement in DJ performance by 16.7\% after 6

376 weeks of plyometric training in competitive distance runners. Although the plyometric exercise

377 volume in both this study and ours was relatively small (60 vs.72 contacts per session), the

378 training appears sufficient in distance runners, who may not be accustomed to such exercises. 
379 Recent research supported that a low-volume plyometric program can produce similar

380 performance improvements in terms of reactive strength compared with that in a high-volume

381 plyometric program (Jeffreys et al. 2017). Taken together, our results support the hypothesis that

382 CT increases neuromuscular facilitation and is appropriate for long-distance runners and may be

383 more efficient than HRT and PLY training methods.

\section{Running economy}

386 Running economy is described as the steady-state oxygen consumption at a given running

387 speed and is a critical physiological measurement to determine distance running success

388 (Saunders et al. 2004). After training intervention, participants in the CT and HRT groups

389 showed a significant improvement in $12 \mathrm{~km} \cdot \mathrm{h}^{-1} \mathrm{RE}(4.47 \mathrm{vs.} 4.11 \%)$ and $14 \mathrm{~km} \cdot \mathrm{h}^{-1} \mathrm{RE}(7.68 \mathrm{vs}$.

$3904.89 \%$ ). Our findings are consistent with those of previous research that reported similar

391 increases in RE after heavy strength interventions for the same level distance runners (Storen et

392 al. 2008). The RE improvement in the CT and HRT groups could be attributed to neuromuscular

393 adaptations after heavy strength training. The multi-joint and closed-chain high load exercises,

394 such as back squat, increases maximum force and peak power, theoretically allowing the athletes

395 to maintain constant speed or to perform each running action at a relatively lower force ratio

396 (Aagaard \& Andersen 2010; Ronnestad \& Mujika 2014). Meanwhile, strength training may

397 potentiate the neural recruitment and function of type I muscle fibers as well as postponing the

398 activation of the less efficient type II fibers (Schumann et al. 2016), therefore reducing overall 
399 energy consumption. In terms of changes in $16 \mathrm{~km} \cdot \mathrm{h}^{-1} \mathrm{RE}$, only the CT group showed a

400 significant improvement (-7.38\%) after training intervention. This may indicate that integrating

401 CT or HRT training into endurance training programs can elicit similar improvements of RE at

402 low speed; as speed increases, the role of CT becomes more substantial. In line with our results,

403 Sedano et al. (Sedano et al. 2013) combined 3 jumping exercises into a strength training program

$404(70 \% 1 \mathrm{RM})$ and showed significantly improvements in well-trained runners' $\mathrm{RE}$ at $16 \mathrm{~km} \cdot \mathrm{h}^{-1}$.

405 Therefore, it can be hypothesized that reactive strength adaptation plays an important role in RE

406 improvement at higher speeds. The elastic energy stored during the eccentric contractions of

407 running and recoiled following concentric contractions makes an extensive contribution to

408 propulsion (Anderson 1996), and may even exceed the efficiency of conversion from chemical

409 energy to kinetic energy by the muscle (Williams 1985). The complex pair exercise in CT may

410 enhance the runners' reactive strength and the ability to store and utilize elastic energy by

411 facilitating the SSC, it theoretically improving the leg stiffness and reducing the time the

412 athletes' foot spends in contact with ground, thereby decreasing the energy cost during running.

413 As running speed increases, elastic mechanisms prevail over the muscle contractile capabilities

414 and may even account for a bigger proportion of the total work(Saunders et al. 2006).

415 In addition, the CT group showed a decrease in BLa concentrations at $16 \mathrm{~km} \cdot \mathrm{h}^{-1}$, which

416 means that athletes could run at the same speed with smaller anaerobic energy consumption. The

417 potential explanation may be that CT combined with endurance training increases the strength

418 and function of type I muscle fibers while delaying the recruitment of type II muscle fibers 
419 (Marcinik et al. 1991; Midgley et al. 2007); thus, reducing lactate production and improving the

420 capability for dispelling lactate at higher running velocities (Jones \& Carter 2000). Therefore,

421 CT can achieve the HRT and PLY training effects at the same time leading to significantly

422 increased RE and decreased BLa concentrations at high speeds.

423

424 Running performance

425 The goal of athletic training is to create meaningful improvements in performance. This

426 study revealed that both the CT and HRT groups showed a significant improvement (-2.8\% vs. -

$4272.09 \%$ ) in 5-km running performance, and the CT group was significantly lower than that in the

428 CON group. The magnitude of the improvement was in line with that in previous studies, which

429 have shown that the increase in running performance was accompanied by augmentation of

430 neuromuscular adaptations and running economy (Berryman et al. 2017; Damasceno et al. 2015;

431 Ramírezcampillo et al. 2014). For example, Ramírezcampillo et al. (Ramírezcampillo et al.

432 2014), demonstrated that jumping and $2.4 \mathrm{~km}$ running performance in highly-trained athletes

433 improved simultaneously after performing 8 weeks of plyometric and endurance training.

434 Damasceno et al. (Damasceno et al. 2015), found that the addition of 8 weeks of heavy strength

435 training to endurance programs resulted in a significant enhancement in $10-\mathrm{km}$ time trials. Since

436 there was no significant change for $\mathrm{VO}_{2} \mathrm{max}$, we speculated that the 5-km performance

437 improvements in the HRT and CT groups were due to the comprehensive effects of

438 neuromuscular adaptations, RE increase, and the decrease in BLa at constant speeds. In addition, 
439 strength and plyometric exercises (back squat, Bulgarian squat and single leg hops, etc.) in the

440 CT group, which involved similar kinematic traits to a running gait, likely provided the greatest

441 improvement in endurance performance (Bazyler et al. 2015). On the other hand, the participants

442 in the CT and HRT groups achieved a significant improvement in their 50-m sprint performance

$443(-2.26 \%$ and $-2.14 \%$ respectively) whereas no significant improvements were found in the CON

444 group. This is in line with that in previous studies, which showed improvements of 1.1-3.4\%

445 following various strength training interventions in distance runners (Mikkola et al. 2007;

446 Paavolainen et al. 1999; Ramírezcampillo et al. 2014). The enhancement of sprint performance

447 plays an important role in distance races and allows runners to hold a favorable position at the

448 start and to accelerate at the end of the race. These findings highlighted the necessity of utilizing

449 a multicomponent conditioning program, such as CT, for developing endurance performance.

450 It is worth noting that the present study only enrolled young male distance runners;

451 therefore, the results may not necessarily be broadly applicable to all types of runners. Since CT

452 was at a relatively high intensity, coaches should be prudent when using this type of training.

453 The age, gender and strength level must be considered before the onset of training. In addition,

454 as HRT has similar training effects on RE and neuromuscular adaptations, coaches could use this

455 type of training to replace the CT when the training subjects are female or older runners. We

456 suggest that a training block of CT could follow a block of HRT so that basic strength levels

457 could be developed. In future studies, we need to investigate the effects of CT on different types

458 of runners (e.g. female or older runners) for longer periods of time $(>16$ weeks) to optimize 
459 endurance performance.

460

\section{Conclusions}

462 In conclusion, we found that compared with strength-endurance training, combining 8

463 weeks of CT or HRT with endurance training resulted in enhanced effects on 1RM strength,

464 CMJ height, running economy, maximal sprint speed and 5-km running performance in well-

465 trained male distance runners. In addition, $\mathrm{CT}$ is an efficient method to combine the advantages

466 of HRT and PLY exercises at the same time, exhibiting greater improvements in terms of

467 reactive strength, $16 \mathrm{~km}^{-1} \mathrm{RE}$, and reduction of BLa concentrations at a speed of $16 \mathrm{~km} \cdot \mathrm{h}^{-1}$.

468 Long-distance runners could integrate CT into their programs to improve the running

469 performance. For well-trained male runners who have limited time to do strength training, three

470 complex-pair exercises three times a week with a relatively small PLY volume, could optimize

471 their endurance performance and is superior to HRT alone.

472

473 Acknowledgements

474 The authors would like to thank all the runners who were involved in this research and all the

475 members and researchers at the Chinese Marathon College, Chinese Athletic Association and

476 Strength and Conditioning Research Center of Shanghai University of Sport for their

477 contribution to this study.

478

479 References

480 Aagaard P, and Andersen JL. 2010. Effects of strength training on endurance capacity in top481 level endurance athletes. Scand J Med Sci Sports 20 Suppl 2:39-47. 10.1111/j.1600- 
482

483

484

485

486

487

488

489

490

491

492

493

494

495

496

497

498

499

500

501

502

503

504

505

506

507

508

509

510

511

512

513

514

515

516

517

518

519

520

0838.2010.01197.x

Anderson T. 1996. Biomechanics and Running Economy. Sports Medicine 22:76-89.

Bazyler, Caleb D, Abbott, Heather A, Bellon, Christopher R, Taber, Christopher B, and Michael H. 2015. Strength Training for Endurance Athletes: Theory to Practice. Strength \& Conditioning Journal 37:1-12.

Beattie K, Carson BP, Lyons M, and Kenny IC. 2016. The Relationship between MaximalStrength and Reactive-Strength. International Journal of Sports Physiology \& Performance 12:548.

Beattie K, Carson BP, Lyons M, Rossiter A, and Kenny IC. 2017. The Effect of Strength Training on Performance Indicators in Distance Runners. J Strength Cond Res 31:9-23. 10.1519/JSC.0000000000001464

Beattie K, Kenny IC, Lyons M, and Carson BP. 2014. The effect of strength training on performance in endurance athletes. Sports Med 44:845-865. 10.1007/s40279-014-0157-y

Berryman N, Mujika I, Arvisais D, Roubeix M, Binet C, and Bosquet L. 2017. Strength Training for Middle- and Long-Distance Performance: A Meta-Analysis. International Journal of Sports Physiology and Performance:1-27. 10.1123/ijspp.2017-0032

Blagrove RC, Howatson G, and Hayes PR. 2018. Use of loaded conditioning activities to potentiate middle- and long-distance performance: a narrative review and practical applications. J Strength Cond Res. 10.1519/JSC.0000000000002456

Carter, Jeremy, Greenwood, and Mike. 2014. Complex Training Reexamined: Review and Recommendations to Improve Strength and Power. Strength \& Conditioning Journal 36:11-19.

Cohen J. 1988. Statistical power analysis for the behavioral sciences. 2nd ed: L. Erlbaum Associates.

Cole AS, Woodruff ME, Horn MP, and Mahon AD. 2006. Strength, Power, and Aerobic Exercise Correlates of 5-km Cross-Country Running Performance in Adolescent Runners. Pediatric Exercise Science 18:págs. 374-384.

Comyns TM, Harrison AJ, Hennessy LK, and Jensen RL. 2006. The optimal complex training rest interval for athletes from anaerobic sports. Journal of Strength \& Conditioning Research 20:471.

Cormie P, Mcguigan MR, and Newton RU. 2011. Developing maximal neuromuscular power: Part 1--biological basis of maximal power production. Sports Medicine 41:17.

Damasceno MV, Lima-Silva AE, Pasqua LA, Tricoli V, Duarte M, Bishop DJ, and Bertuzzi R. 2015. Effects of resistance training on neuromuscular characteristics and pacing during 10-km running time trial. Eur J Appl Physiol 115:1513-1522. 10.1007/s00421-015-3130$\mathrm{Z}$

Díaz JJ, Fernández-Ozcorta EJ, and Santos-Concejero J. 2018. The influence of pacing strategy on marathon world records. European Journal of Sport Science:1.

Docherty D, and Sporer B. 2000. A proposed model for examining the interference phenomenon 
521

522

523

524

525

526

527

528

529

530

531

532

533

534

535

536

537

538

539

540

541

542

543

544

545

546

547

548

549

550

551

552

553

554

555

556

557

558

559

between concurrent aerobic and strength training. Sports Medicine 30:385-394.

Esformes JI, and Bampouras TM. 2013. Effect of back squat depth on lower-body postactivation potentiation. Journal of Strength and Conditioning Research 27:2997-3000.

Jeffreys M, De SCM, Lloyd RS, Oliver JL, and Hughes J. 2017. The effect of varying plyometric volume on stretch-shortening cycle capability in collegiate male rugby players. Journal of Strength \& Conditioning Research.

Jones AM, and Carter H. 2000. The Effect of Endurance Training on Parameters of Aerobic Fitness. Sports Medicine 29:373.

Jones P, and Lees A. 2003. A biomechanical analysis of the acute effects of complex training using lower limb exercises. Journal of Strength \& Conditioning Research 17:694-700.

Macdonald CJ, Lamont HS, and Garner JC. 2012. A comparison of the effects of 6 weeks of traditional resistance training, plyometric training, and complex training on measures of strength and anthropometrics. Journal of Strength \& Conditioning Research 26:422-431.

Maio Alves JM, Rebelo AN, Abrantes C, Sampaio J, and Sampaio J. 2010. Short-term effects of complex and contrast training in soccer players' vertical jump, sprint, and agility abilities. Journal of Strength \& Conditioning Research 24:936.

Marcinik EJ, Potts J, Schlabach G, Will S, Dawson P, and Hurley BF. 1991. Effects of strength training on lactate threshold and endurance performance. Medicine \& Science in Sports \& Exercise 23:739.

MCBRIDE, Jeffrey M, TRIPLETTMCBRIDE, TRAVIS, DAVIE, ALLAN, and Robert U. 1999. A Comparison of Strength and Power Characteristics Between Power Lifters, Olympic Lifters, and Sprinters. Journal of Strength \& Conditioning Research 13:58-66.

Midgley AW, McNaughton LR, and Jones AM. 2007. Training to Enhance the Physiological Determinants of Long-Distance Running Performance. Sports Medicine 37:857-880. 10.2165/00007256-200737100-00003

Mikkola J, Rusko H, Nummela A, Pollari T, and Hakkinen K. 2007. Concurrent endurance and explosive type strength training improves neuromuscular and anaerobic characteristics in young distance runners. Int J Sports Med 28:602-611. 10.1055/s-2007-964849

Nummela AT, Paavolainen LM, Sharwood KA, Lambert MI, Noakes TD, and Rusko HK. 2006. Neuromuscular factors determining $5 \mathrm{~km}$ running performance and running economy in well-trained athletes. Eur J Appl Physiol 97:1-8. 10.1007/s00421-006-0147-3

Paavolainen L, Nummela A, and Rusko H. 2000. Muscle power factors and VO2max as determinants of horizontal and uphill running performance. Scandinavian Journal of Medicine \& Science in Sports 10:286-291.

Paavolainen LM, Nummela AT, and Rusko HK. 1999. Neuromuscular characteristics and muscle power as determinants of 5-km running performance. Med Sci Sports Exerc 31:124-130.

Piacentini MF, De IG, Comotto S, Spedicato A, Vernillo G, and La TA. 2013. Concurrent strength and endurance training effects on running economy in master endurance runners. 
560

561

562

563

564

565

566

567

568

569

570

571

572

573

574

575

576

577

578

579

580

581

582

583

584

585

586

587

588

589

590

591

592

593

594

595

596

597

598

Journal of Strength \& Conditioning Research 27:2295.

Ramírezcampillo R, Alvarez C, Henríquezolguín C, Baez EB, Martínez C, Andrade DC, and Izquierdo M. 2014. Effects of plyometric training on endurance and explosive strength performance in competitive middle- and long-distance runners. Journal of Strength \& Conditioning Research 28:97.

Ronnestad BR, and Mujika I. 2014. Optimizing strength training for running and cycling endurance performance: A review. Scand J Med Sci Sports 24:603-612. 10.1111/sms. 12104

Santos EJ, and Janeira MA. 2008. Effects of complex training on explosive strength in adolescent male basketball players. Journal of Strength \& Conditioning Research 22:903-909.

Saunders PU, Pyne DB, Telford RD, and Hawley JA. 2004. Factors affecting running economy in trained distance runners. Sports Medicine 34:465-485.

Saunders PU, Telford RD, Pyne DB, Peltola EM, Cunningham RB, Gore CJ, and Hawley JA. 2006. Short-term plyometric training improves running economy in highly trained middle and long distance runners. Journal of Strength \& Conditioning Research 20:947.

Schumann M, Pelttari P, Doma K, Karavirta L, and Hakkinen K. 2016. Neuromuscular Adaptations to Same-Session Combined Endurance and Strength Training in Recreational Endurance Runners. Int J Sports Med 37:1136-1143. 10.1055/s-0042112592

Sedano S, Marín PJ, Cuadrado G, and Redondo JC. 2013. Concurrent training in elite male runners: the influence of strength versus muscular endurance training on performance outcomes. Journal of Strength \& Conditioning Research 27:2433.

Spurrs RW, Murphy AJ, and Watsford ML. 2003. The effect of plyometric training on distance running performance. Eur J Appl Physiol 89:1-7. 10.1007/s00421-002-0741-y

Stone MH, Stone ME, and Sands WA. 2006. Maximum Strength and Strength Training---A Relationship to Endurance? Strength \& Conditioning Journal 28:págs. 44-53.

Storen O, Helgerud J, Stoa EM, and Hoff J. 2008. Maximal strength training improves running economy in distance runners. Med Sci Sports Exerc 40:1087-1092. 10.1249/MSS.0b013e318168da2f

Suchomel TJ, Nimphius S, and Stone MH. 2016. The Importance of Muscular Strength in Athletic Performance. Sports Med 46:1419-1449. 10.1007/s40279-016-0486-0

Tillin NA, and Bishop D. 2009. Factors modulating post-activation potentiation and its effect on performance of subsequent explosive activities. Sports Medicine 39:147-166.

Vikmoen O, Raastad T, Seynnes O, Bergstrom K, Ellefsen S, and Ronnestad BR. 2016. Effects of Heavy Strength Training on Running Performance and Determinants of Running Performance in Female Endurance Athletes. PLoS One 11:0150799. 10.1371/journal.pone.0150799

Williams KR. 1985. The relationship between mechanical and physiological energy estimates. 


\section{Table 1 (on next page)}

Baseline characteristics of subjects in each training group (Mean \pm SD) 


\begin{tabular}{|c|c|c|c|c|c|}
\hline & $\begin{array}{l}\text { Complex Training Group } \\
\qquad(\mathrm{N}=10)\end{array}$ & $\begin{array}{l}\text { Heavy resistance Group } \\
\qquad(\mathrm{N}=9)\end{array}$ & $\begin{array}{l}\text { Control Group } \\
\qquad(\mathrm{N}=9)\end{array}$ & F value & $\mathrm{p}$ value \\
\hline Age (years) & $20.2 \pm 1.03$ & $21.22 \pm 1.48$ & $20.78 \pm 1.20$ & 1.616 & 0.219 \\
\hline Height (cm) & $178.2 \pm 6.1$ & $175.3 \pm 3.8$ & $178.5 \pm 4.4$ & 1.146 & 0.334 \\
\hline $\mathrm{BM}(\mathrm{kg})$ & $63.08 \pm 6.08$ & $57.76 \pm 3.70$ & $61.36 \pm 4.49$ & 2.863 & 0.076 \\
\hline $\operatorname{BMI}\left(\mathrm{kg} \cdot \mathrm{m}^{-2}\right)$ & $19.86 \pm 1.61$ & $18.77 \pm 1.03$ & $19.24 \pm 0.81$ & 1.850 & 0.178 \\
\hline FFM $(\mathrm{kg})$ & $55.99 \pm 4.86$ & $52.26 \pm 3.04$ & $55.97 \pm 4.02$ & 2.545 & 0.099 \\
\hline $\mathrm{FM}(\mathrm{kg})$ & $7.09 \pm 3.14$ & $5.50 \pm 1.99$ & $5.39 \pm 1.70$ & 1.516 & 0.239 \\
\hline $\mathrm{VO}_{2} \max \left(\mathrm{ml} \cdot \mathrm{kg} \cdot \mathrm{min}^{-1}\right)$ & $65.65 \pm 5.06$ & $65.54 \pm 5.52$ & $66.14 \pm 5.25$ & 0.033 & 0.968 \\
\hline $5-\mathrm{km}$ time $(\mathrm{s})$ & $953.70 \pm 12.30$ & $952.56 \pm 10.10$ & $954.11 \pm 6.75$ & 0.058 & 0.944 \\
\hline
\end{tabular}

BM, body mass; BMI, body mass index; FFM, fat-free mass; FM, fat mass; $\mathrm{VO}_{2}$ max, maximum oxygen uptake 
Table 2 (on next page)

Training arrangement during 8-week intervention 


\begin{tabular}{|c|c|c|c|c|c|c|c|}
\hline & Monday & Tuesday & Wednesday & Thursday & Friday & Saturday & Sunday \\
\hline \multirow[t]{4}{*}{ Moring } & Road running: $15-$ & IT: $1000 \mathrm{~m} * 5$ sets $(90-$ & Road running: $15-$ & IT:1000m*5sets $(90-$ & Road running: $15-$ & IT:1000m*5sets $(90-$ & Rest \\
\hline & $20 \mathrm{~km}(75-$ & $95 \% \mathrm{HR}_{\mathrm{MAX}}$ ), work rest & $20 \mathrm{~km}\left(75-85 \% \mathrm{HR}_{\mathrm{MAX}}\right)$ & $95 \% \mathrm{HR}_{\mathrm{MAX}}$ ), work rest & $20 \mathrm{~km}\left(75-85 \% \mathrm{HR}_{\text {MAX }}\right)$ & $95 \% \mathrm{HR}_{\mathrm{MAX}}$ ), work rest ratio $1: 1$ & \\
\hline & $\left.85 \% \mathrm{HR}_{\mathrm{MAX}}\right)$ & ratio $1: 1$ & & ratio $1: 1$ & & Road running: $10 \mathrm{~km}(70-$ & \\
\hline & & & & & & $\left.80 \% \mathrm{HR}_{\mathrm{MAX}}\right)$ & \\
\hline Afternoon & Strength training & & Strength training & & Strength training & & \\
\hline
\end{tabular}

$\mathrm{HR}_{\mathrm{MAX}}$, Maximal heart rate; IT, Interval training 
Table 3 (on next page)

Strength training program during study 


\begin{tabular}{|c|c|c|c|c|}
\hline Exercise & Load & Sets & Repetition & Recovery(mins) \\
\hline \multicolumn{5}{|c|}{ Preparatory Strength Training } \\
\hline Back squat & $20 \mathrm{~kg}$ & 3 & 10 & 3 \\
\hline Bulgarian squat & $20 \mathrm{~kg}$ & 3 & $5 \mathrm{ES}$ & 3 \\
\hline Romanian deadlifts & $20 \mathrm{~kg}$ & 3 & 10 & 3 \\
\hline \multicolumn{5}{|l|}{ Training intervention } \\
\hline \multicolumn{5}{|l|}{ CT group } \\
\hline Back squat & $80-85 \% 1 \mathrm{RM}$ & 3 & 5 & 4 \\
\hline Drop jump & 40-cm Box & 3 & 6 & 4 \\
\hline Bulgarian squat & $80-85 \% 1 \mathrm{RM}$ & 3 & $5 \mathrm{ES}$ & 4 \\
\hline Single leg hop & BW & 3 & $6 \mathrm{ES}$ & 4 \\
\hline Romanian deadlifts & $80-85 \% 1 \mathrm{RM}$ & 3 & 5 & 4 \\
\hline Double leg hurdle hop & $50 \mathrm{~cm}$ Hurdle & 3 & 6 & 4 \\
\hline \multicolumn{5}{|l|}{ HRT group } \\
\hline Back squat & $80-85 \% 1 \mathrm{RM}$ & 5 & 5 & 3 \\
\hline Bulgarian squat & $80-85 \% 1 \mathrm{RM}$ & 5 & $5 \mathrm{ES}$ & 3 \\
\hline Romanian deadlift & $80-85 \% 1 \mathrm{RM}$ & 5 & 5 & 3 \\
\hline \multicolumn{5}{|l|}{ CON group } \\
\hline Back squat & $40 \% 1 \mathrm{RM}$ & 5 & 20 & 1 \\
\hline Bulgarian squat & $40 \% 1 \mathrm{RM}$ & 5 & $15 \mathrm{ES}$ & 1 \\
\hline Romanian deadlift & $40 \% 1 \mathrm{RM}$ & 5 & 20 & 1 \\
\hline
\end{tabular}

33

34 RM, maximal repetition; BW, body weight; ES, each side 
Table 4 (on next page)

Results of body composition before and after intervention (Mean \pm SD) 


\begin{tabular}{|c|c|c|c|c|c|c|c|c|c|}
\hline & \multicolumn{3}{|c|}{ Complex Training Group $(\mathrm{N}=10)$} & \multicolumn{3}{|c|}{ Heavy resistance Group $(\mathrm{N}=9)$} & \multicolumn{3}{|c|}{ Control Group (N=9) } \\
\hline & Before & After & $\% \Delta$ & Before & After & $\% \Delta$ & Before & After & $\% \Delta$ \\
\hline $\mathrm{BM}$ & $63.08 \pm 6.08$ & $63.44 \pm 6.85$ & $0.53 \pm 3.71$ & $57.76 \pm 3.70$ & $57.92 \pm 3.05$ & $0.39 \pm 2.64$ & $61.36 \pm 4.49$ & $61.27 \pm 4.69$ & $-0.16 \pm 0.97$ \\
\hline \multicolumn{10}{|l|}{$(\mathrm{kg})$} \\
\hline BMI & $19.86 \pm 1.61$ & $19.84 \pm 1.66$ & $-0.03 \pm 4.33$ & $18.77 \pm 1.03$ & $18.86 \pm 0.86$ & $0.43 \pm 2.46$ & $19.24 \pm 0.81$ & $19.27 \pm 0.95$ & $0.10 \pm 1.54$ \\
\hline \multicolumn{10}{|c|}{$\left(\mathrm{kg} \cdot \mathrm{m}^{-2}\right)$} \\
\hline FFM & $55.99 \pm 4.86$ & $56.39 \pm 5.39$ & $0.66 \pm 1.86$ & $52.26 \pm 3.04$ & $52.10 \pm 2.85$ & $-0.24 \pm 2.90$ & $55.97 \pm 4.02$ & $55.74 \pm 4.10$ & $-0.40 \pm 0.82$ \\
\hline \multicolumn{10}{|l|}{$(\mathrm{kg})$} \\
\hline FM & $7.09 \pm 3.14$ & $7.05 \pm 3.16$ & $3.88 \pm 6.29$ & $5.50 \pm 1.99$ & $5.82 \pm 2.13$ & $6.69 \pm 13.52$ & $5.39 \pm 1.70$ & $5.52 \pm 1.95$ & $1.60 \pm 7.92$ \\
\hline$(\mathrm{kg})$ & & & & & & & & & \\
\hline
\end{tabular}

BM, body mass; BMI, body mass index; FFM, fat-free mass; FM, fat mass. 


\section{Table 5 (on next page)}

Results of strength and power assessment before and after intervention (Mean \pm SD) 


\begin{tabular}{|c|c|c|c|c|c|c|c|c|c|}
\hline & \multicolumn{3}{|c|}{ Complex Training Group $(\mathrm{N}=10)$} & \multicolumn{3}{|c|}{ Heavy resistance Group $(\mathrm{N}=9)$} & \multicolumn{3}{|c|}{ Control Group (N=9) } \\
\hline & Before & After & $\% \Delta$ & Before & After & $\% \Delta$ & Before & After & $\% \Delta$ \\
\hline $1 \mathrm{RM}$ & $60.25 \pm 8.03$ & $70.50 \pm 11.17 * * *$ & $16.88 \pm 5.93$ & $60.56 \pm 11.84$ & $71.67 \pm 12.50^{* * *}$ & $18.80 \pm 6.42$ & $63.33 \pm 9.35$ & $64.44 \pm 8.82$ & $2.15 \pm 6.64$ \\
\hline \multicolumn{10}{|l|}{ (kg) } \\
\hline CMJ Height & $31.06 \pm 3.41$ & $34.51 \pm 3.85^{* * *}$ & $11.28 \pm 7.57$ & $32.8 \pm 4.23$ & $35.58 \pm 3.33 * * *$ & $8.96 \pm 4.94$ & $33.46 \pm 4.27$ & $34.26 \pm 4.22$ & $2.46 \pm 1.64$ \\
\hline \multicolumn{10}{|l|}{$(\mathrm{cm})$} \\
\hline Peak Power & $43.66 \pm 2.70$ & $47.12 \pm 2.65$ & $8.20 \pm 7.79$ & $45.18 \pm 3.57$ & $47.45 \pm 5.33$ & $5.05 \pm 8.69$ & $45.08 \pm 3.17$ & $45.29 \pm 3.01$ & $0.53 \pm 3.66$ \\
\hline \multicolumn{10}{|l|}{$(w / k g)$} \\
\hline DJ Height & $31.39 \pm 4.41$ & $35.38 \pm 4.55^{* * *}$ & $12.94 \pm 4.67$ & $33.03 \pm 3.79$ & $33.96 \pm 4.13$ & $2.88 \pm 6.58$ & $33.31 \pm 4.53$ & $32.81 \pm 4.26$ & $-1.40 \pm 1.74$ \\
\hline \multicolumn{10}{|l|}{$(\mathrm{cm})$} \\
\hline RSI & $59.05 \pm 11.63$ & $70.80 \pm 15.69^{* * *}$ & $19.99 \pm 12.39$ & $61.57 \pm 12.95$ & $66.71 \pm 8.53$ & $10.43 \pm 15.69$ & $62.11 \pm 12.04$ & $62.91 \pm 12.07$ & $1.41 \pm 5.70$ \\
\hline$(\mathrm{cm} / \mathrm{s})$ & & & & & & & & & \\
\hline
\end{tabular}

1RM, one repetition maximum back squat; CMJ, counter movement jump height; DJ, drop jump height; Peak Power, peak power in counter movement jump; RSI, reactive strength index. ${ }^{*} \mathrm{p}<0.0167, * * \mathrm{p}<0.01, * * * \mathrm{p}<0.001$ significant difference from pre- to post-test. 
Table 6(on next page)

Results of physiological and running performance assessment before and after intervention (Mean \pm SD) 


\begin{tabular}{|c|c|c|c|c|c|c|c|c|c|}
\hline & \multicolumn{3}{|c|}{ Complex Training Group $(\mathrm{N}=10)$} & \multicolumn{3}{|c|}{ Heavy resistance Group $(\mathrm{N}=9)$} & \multicolumn{3}{|c|}{ Control Group $(\mathrm{N}=9)$} \\
\hline & Before & After & $\% \Delta$ & Before & After & $\% \Delta$ & Before & After & $\% \Delta$ \\
\hline $\mathrm{VO}_{2} \max$ & $65.65 \pm 5.06$ & $64.47 \pm 4.31$ & $-1.57 \pm 5.78$ & $65.54 \pm 5.52$ & $64.65 \pm 6.18$ & $-1.25 \pm 6.58$ & $66.14 \pm 5.25$ & $67.79 \pm 3.03$ & $2.81 \pm 5.05$ \\
\hline \multicolumn{10}{|l|}{$\left(\mathrm{ml} \cdot \mathrm{kg} \cdot \mathrm{min}^{-1}\right)$} \\
\hline $\mathrm{RE}$ at $12 \mathrm{~km} \cdot \mathrm{h}^{-1}$ & $46.62 \pm 3.20$ & $44.42 \pm 2.32 * *$ & $-4.47 \pm 5.92$ & $47.01 \pm 3.14$ & $45.06 \pm 3.82 *$ & $-4.11 \pm 5.33$ & $47.81 \pm 3.24$ & $45.71 \pm 3.05^{*}$ & $-4.38 \pm 1.59$ \\
\hline \multicolumn{10}{|l|}{$\left(\mathrm{ml} \cdot \mathrm{kg} \cdot \mathrm{min}^{-1}\right)$} \\
\hline $\mathrm{RE}$ at $14 \mathrm{~km} \cdot \mathrm{h}^{-1}$ & $54.45 \pm 3.18$ & $50.17 \pm 3.04 * * *$ & $-7.68 \pm 6.33$ & $53.41 \pm 3.26$ & $50.78 \pm 4.07 * *$ & $-4.89 \pm 5.26$ & $53.95 \pm 2.27$ & $52.33 \pm 2.31$ & $-2.98 \pm 2.62$ \\
\hline \multicolumn{10}{|l|}{$\left(\mathrm{ml} \cdot \mathrm{kg} \cdot \mathrm{min}^{-1}\right)$} \\
\hline $\mathrm{RE}$ at $16 \mathrm{~km} \cdot \mathrm{h}^{-1}$ & $60.77 \pm 4.17$ & $56.09 \pm 2.55^{* * *}$ & $-7.38 \pm 6.67$ & $59.60 \pm 3.04$ & $57.88 \pm 4.11$ & $-2.91 \pm 4.21$ & $61.00 \pm 3.00$ & $61.35 \pm 1.79$ & $0.72 \pm 3.99$ \\
\hline \multicolumn{10}{|l|}{$\left(\mathrm{ml} \cdot \mathrm{kg} \cdot \mathrm{min}^{-1}\right)$} \\
\hline BLa at $12 \mathrm{~km} \cdot \mathrm{h}^{-1}$ & $1.46 \pm 0.43$ & $1.29 \pm 0.31$ & $-1.93 \pm 43.93$ & $1.44 \pm 0.46$ & $1.24 \pm 0.40$ & $-12.72 \pm 16.13$ & $1.43 \pm 0.53$ & $1.37 \pm 0.39$ & $2.60 \pm 30.62$ \\
\hline \multicolumn{10}{|l|}{$\left(\mathrm{mmol} \cdot \mathrm{L}^{-1}\right)$} \\
\hline BLa at $14 \mathrm{~km} \cdot \mathrm{h}^{-1}$ & $1.60 \pm 0.63$ & $1.29 \pm 0.43$ & $-12.50 \pm 30.80$ & $1.62 \pm 0.73$ & $1.48 \pm 0.53$ & $1.12 \pm 37.82$ & $1.67 \pm 0.35$ & $1.32 \pm 0.36$ & $45.06 \pm 56.99$ \\
\hline \multicolumn{10}{|l|}{$\left(\mathrm{mmol} \cdot \mathrm{L}^{-1}\right)$} \\
\hline BLa at $16 \mathrm{~km} \cdot \mathrm{h}^{-1}$ & $2.56 \pm 0.48$ & $1.45 \pm 0.45^{* * *}$ & $-40.80 \pm 14.82$ & $2.30 \pm 0.53$ & $1.94 \pm 0.68$ & $-16.05 \pm 17.89$ & $2.76 \pm 0.59$ & $2.41 \pm 0.51$ & $-8.09 \pm 32.15$ \\
\hline \multicolumn{10}{|l|}{$\left(\mathrm{mmol} \cdot \mathrm{L}^{-1}\right)$} \\
\hline $\mathrm{HR}$ at $12 \mathrm{~km} \cdot \mathrm{h}^{-1}$ & $136.34 \pm 7.97$ & $136.14 \pm 8.80$ & $-0.07 \pm 5.00$ & $138.86 \pm 12.92$ & $136.54 \pm 8.74$ & $-1.29 \pm 6.19$ & $142.07 \pm 13.59$ & $143.54 \pm 15.12$ & $1.10 \pm 6.21$ \\
\hline
\end{tabular}




\begin{tabular}{|c|c|c|c|c|c|c|c|c|c|}
\hline $\mathrm{HR}$ at $14 \mathrm{~km} \cdot \mathrm{h}^{-1}$ & $149.08 \pm 8.60$ & $149.28 \pm 9.32$ & $0.18 \pm 4.13$ & $153.13 \pm 14.41$ & $149.16 \pm 9.84$ & $-2.29 \pm 4.86$ & $155.34 \pm 13.22$ & $156.03 \pm 14.83$ & $0.53 \pm 6.34$ \\
\hline \multicolumn{10}{|l|}{$\left(\right.$ beats $\cdot \min ^{-1}$ ) } \\
\hline $\mathrm{HR}$ at $16 \mathrm{~km} \cdot \mathrm{h}^{-1}$ & $164.81 \pm 8.31$ & $160.74 \pm 7.51$ & $-2.43 \pm 1.83$ & $166.95 \pm 13.77$ & $164.41 \pm 10.39$ & $-1.35 \pm 3.71$ & $169.59 \pm 14.78$ & $169.93 \pm 16.33$ & $0.31 \pm 6.72$ \\
\hline \multicolumn{10}{|l|}{$\left(\right.$ beats $\cdot \min ^{-1}$ ) } \\
\hline 5-km time (s) & $953.70 \pm 12.30$ & $926.90 \pm 9.92 * * * \# \#$ & $-2.80 \pm 0.87$ & $952.56 \pm 10.10$ & $932.67 \pm 11.61 * * *$ & $-2.09 \pm 0.52$ & $954.11 \pm 6.75$ & $947.33 \pm 10.03$ & $-0.07 \pm 1.51$ \\
\hline 50-m time (s) & $6.25 \pm 0.19$ & $6.11 \pm 0.24 * *$ & $-2.26 \pm 2.16$ & $6.17 \pm 0.33$ & $6.04 \pm 0.33 * *$ & $-2.14 \pm 2.35$ & $5.94 \pm 0.21$ & $5.92 \pm 0.30$ & $-0.4 \pm 2.03$ \\
\hline
\end{tabular}

BLa, blood lactate concentrations; $\mathrm{HR}$, heart rate; RE, running economy; $\mathrm{VO}_{2}$ max, maximum oxygen uptake. ${ }^{*} \mathrm{p}<0.0167,{ }^{* *} \mathrm{p}<0.01, * * * \mathrm{p}<0.001$ significant difference from preto post-test; \#\#p<0.01, significantly different from control group at post-test. 
Figure 1

Flowchart of the study design.

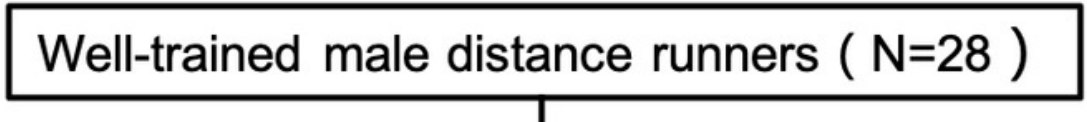

\section{2-week preparatory strength training}

Correct strength technique

Familiarize the test procedure

\section{Pre-test (two-day test protocol)}

1st Day: Body composition; 1RM strength, CMJ and DJ; 2nd Day: Running economy; $\mathrm{VO}_{2}$ max; 50-m and 5-km running performance

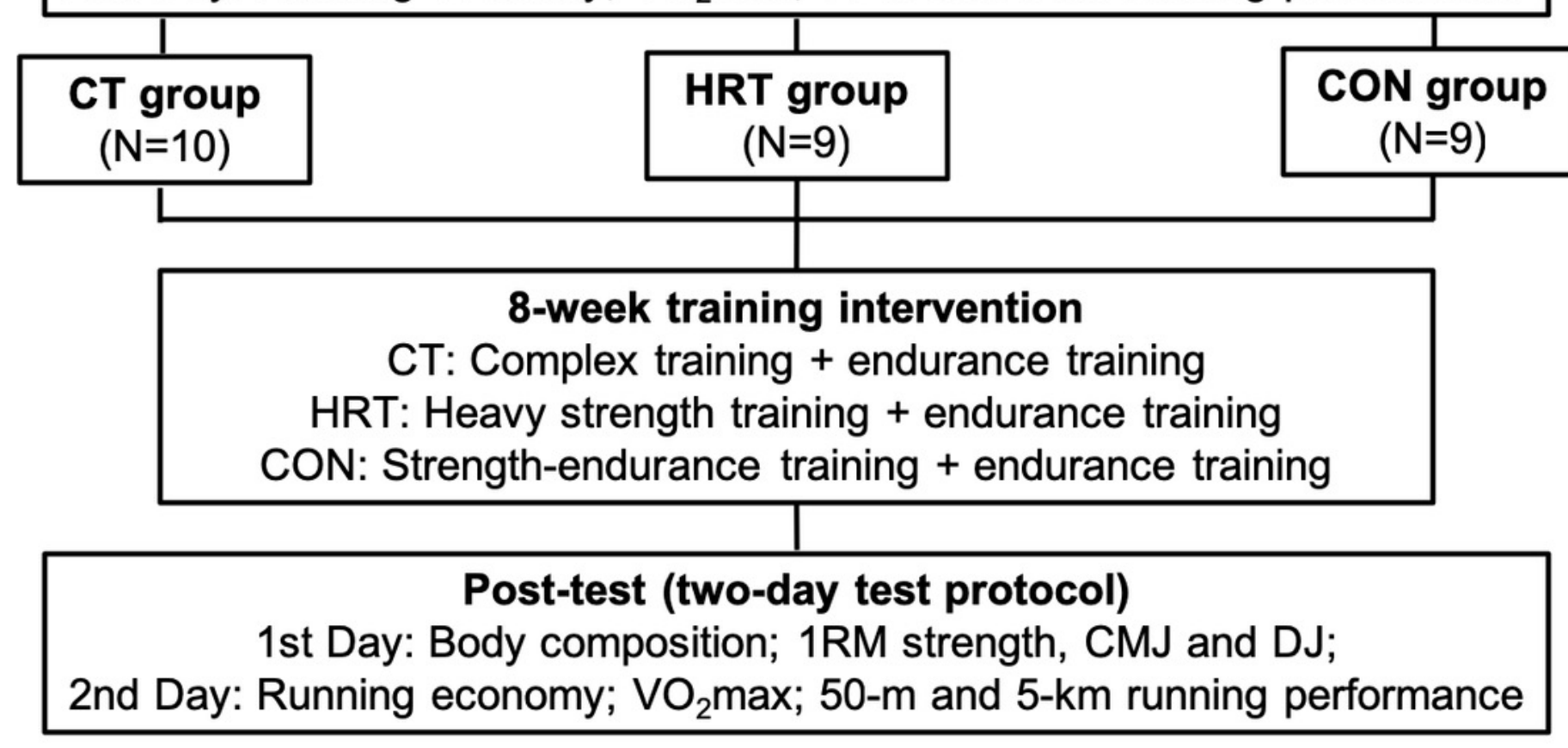


Figure 2 (on next page)

Neuromuscular adaptation over 8 weeks of intervention

1RM (A), CMJ (B), DJ (C) and RSI (D) over 8 weeks of intervention (Mean $\pm S D)$. 1RM, one repetition maximum strength; CMJ, counter movement jump height; DJ, drop jump height; RSI, reactive strength index. ${ }^{* * *} p<0.001$ significant difference from pre- to post-test. 

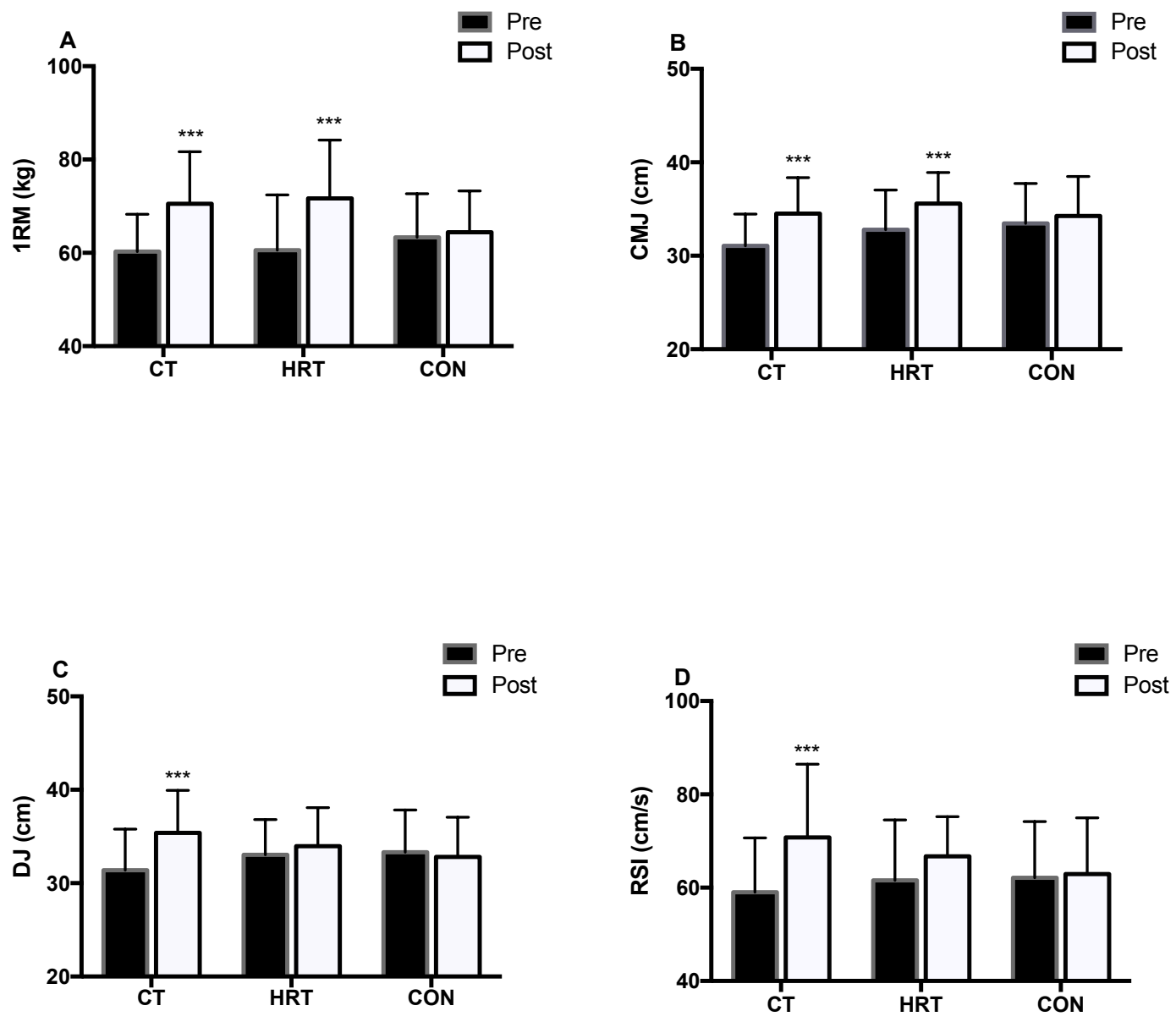
Figure 3 (on next page)

Running economy changes over 8 weeks of intervention

Running economy at (A) 12 , (B) 14 and (C) $16 \mathrm{~km} \cdot \mathrm{h}^{-1}$ over 8 weeks of intervention (Mean $\pm S D$ ). $* p<0.0167, * * p<0.01, * * p<0.001$ significant difference from pre- to post-test. 


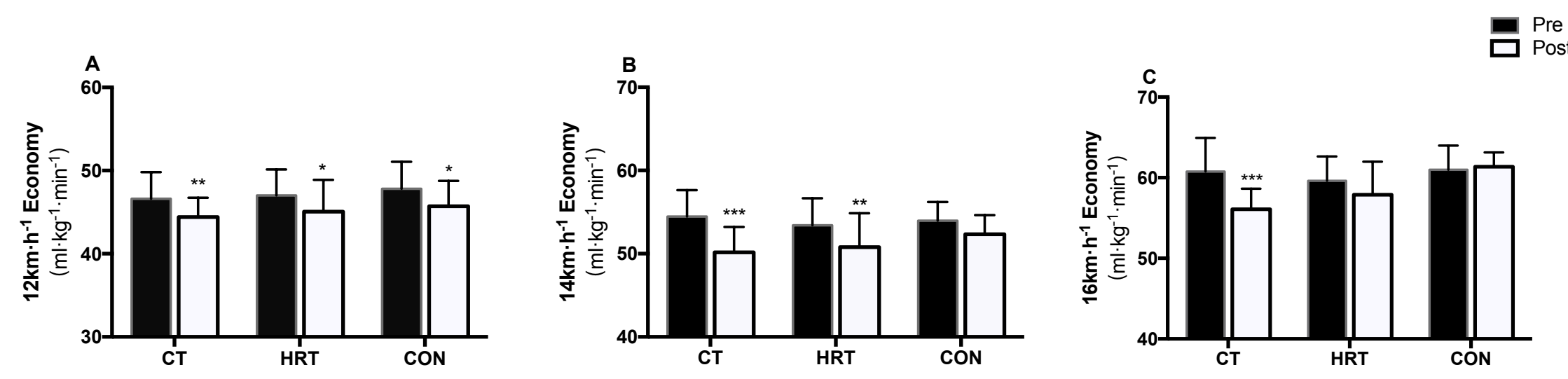


Figure 4 (on next page)

Blood lactate concentrations changes over 8 weeks of intervention

Blood lactate concentrations at the speeds of $12,14,16 \mathrm{~km} \cdot \mathrm{h}^{-1}$ over 8 weeks of intervention (Mean $\pm \mathrm{SD}$ ). ${ }^{* * *} p<0.001$, significant difference from pre- to post-test. 

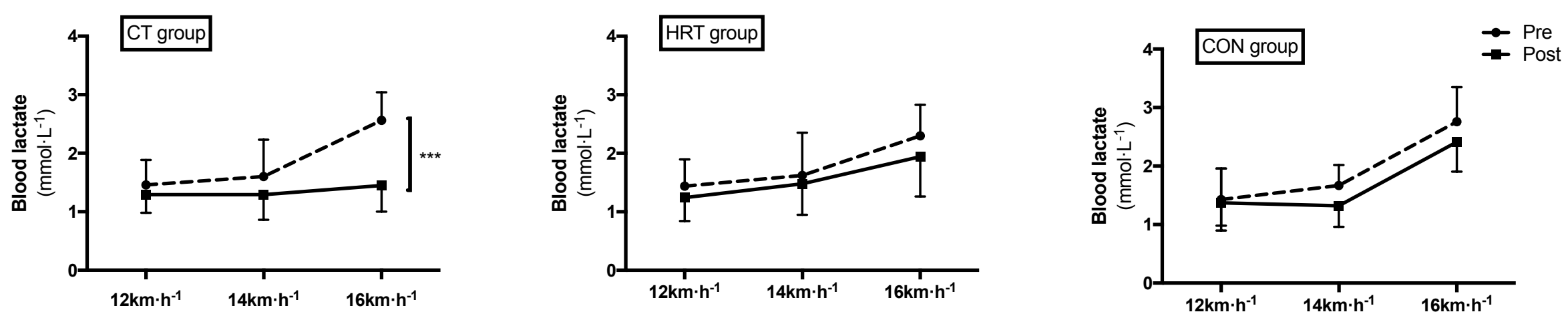
Figure $\mathbf{5}$ (on next page)

5-km running and 50-m sprint performance changes over 8 weeks of intervention

5-km running (A) and 50-m sprint (B) performance over 8 weeks of intervention (Mean $\pm S D$ ). ${ }^{* *} p<0.01,{ }^{* * *} p<0.001$, significant difference from pre- to post-test; $\# \# p<0.01$, significant difference from CON group. 

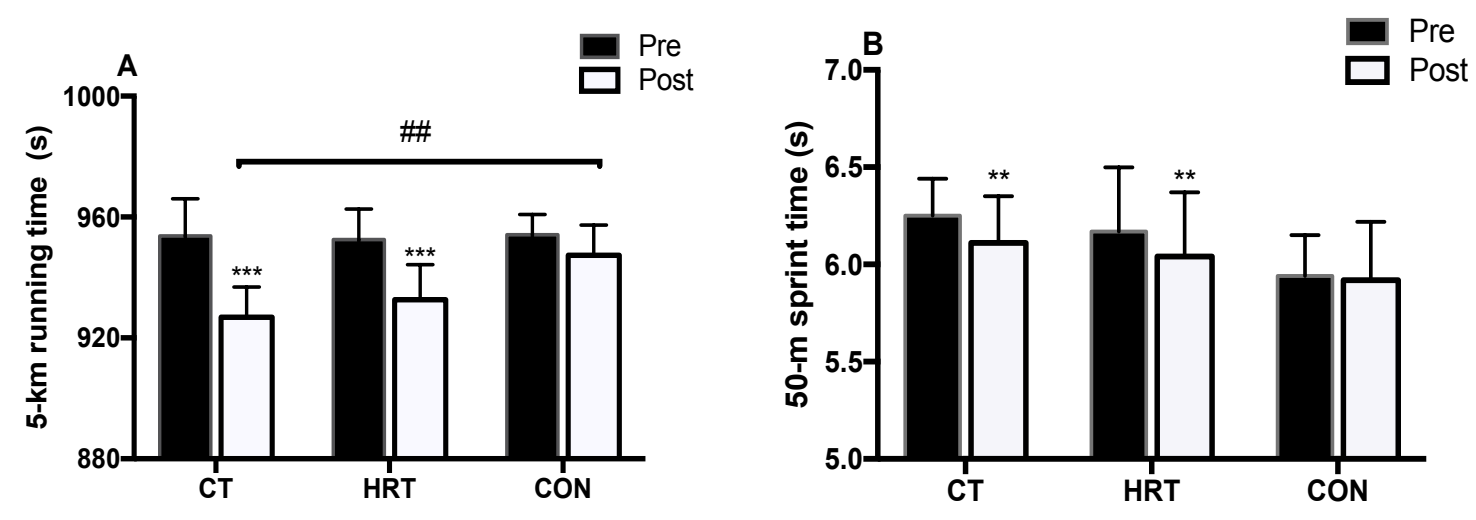